\title{
ARTICLE
}

Multiple myeloma gammopathies

\section{Integrated safety profile of selinexor in multiple myeloma: experience from 437 patients enrolled in clinical trials}

\author{
Maria Gavriatopoulou ${ }^{1}$ - Ajai Chari ${ }^{2}$ - Christine $\mathrm{Chen}^{3}$ - Nizar Bahlis ${ }^{4}$ - Dan T. Vogl $\mathbb{1}^{5}$ - Andrzej Jakubowiak ${ }^{6}$. \\ David Dingli ${ }^{7}$ - Robert F. Cornell ${ }^{8}$. Craig C. Hofmeister $\mathbb{D}^{9} \cdot{\text { David Siegel } \mathbb{D}^{10} \text { - Jesus G. Berdeja }}^{11}$. Donna Reece ${ }^{3}$. \\ Darrell White ${ }^{12}$. Suzanne Lentzsch ${ }^{13} \cdot$ Cristina Gasparetto $^{14}$. Carol Ann Huff ${ }^{15}$. Sundar Jagannath ${ }^{2} \cdot$ Rachid Baz $^{16}$. \\ Ajay K. Nooka ${ }^{17}{ }^{17} \cdot$ Joshua Richter $^{10} \cdot$ Rafat Abonour $^{18} \cdot$ Terri L. Parker $^{19}$ - Andrew J. Yee ${ }^{20}$. Philippe Moreau ${ }^{21}$. \\ Sagar Lonial ${ }^{17}$ - Sascha Tuchman ${ }^{22} \cdot$ Katja C. Weisel $^{23} \cdot$ Mohamad Mohty $^{24} \cdot$ Sylvain Choquet $^{25} \cdot$ T. J. Unger ${ }^{26}$. \\ Kai $\mathrm{Li}^{26} \cdot \mathrm{Yi} \mathrm{Chai}^{26} \cdot$ Lingling $\mathrm{Li}^{26}$ - Jatin Shah ${ }^{26}$. Sharon Shacham ${ }^{26}$ - Michael G. Kauffman ${ }^{26}$. \\ Meletios Athanasios Dimopoulos'
}

Received: 15 October 2019 / Revised: 7 February 2020 / Accepted: 10 February 2020 / Published online: 24 February 2020

(c) The Author(s) 2020. This article is published with open access

\begin{abstract}
Selinexor is an oral, small molecule inhibitor of the nuclear export protein exportin 1 with demonstrated activity in hematologic and solid malignancies. Side effects associated with selinexor include nausea, vomiting, fatigue, diarrhea, decreased appetite, weight loss, thrombocytopenia, neutropenia, and hyponatremia. We reviewed 437 patients with multiple myeloma treated with selinexor and assessed the kinetics of adverse events and impact of supportive care measures. Selinexor reduced both platelets and neutrophils over the first cycle of treatment and reached a nadir between 28 and 42 days. Platelet transfusions and thrombopoietin receptor agonists were effective at treating thrombocytopenia, and granulocyte colony stimulating factors were effective at resolving neutropenia. The onset of gastrointestinal side effects (nausea, vomiting, and diarrhea) was most common during the first 1-2 weeks of treatment. Nausea could be mitigated with 5-HT3 antagonists and either neurokinin 1 receptor antagonists, olanzapine, or cannbainoids. Loperamide and bismuth subsalicylate ameliorated diarrhea. The primary constitutional side effects of fatigue and decreased appetite could be managed with methylphenidate, megestrol, cannabinoids or olanzapine, respectively. Hyponatremia was highly responsive to sodium replacement. Selinexor has well-established adverse effects that mainly occur within the first 8 weeks of treatment, are reversible, and respond to supportive care.
\end{abstract}

\section{Introduction}

Multiple myeloma (MM) is characterized by the aberrant, clonal expansion of malignant plasma cells, resulting in hypercalcemia, renal impairment, anemia, bone lesions, and susceptibility to infections. It is estimated that 138,500 people are living with myeloma worldwide and over 98,400 will succumb to the disease this year [1]. Though MM remains largely incurable, advances in autologous

Supplementary information The online version of this article (https:// doi.org/10.1038/s41375-020-0756-6) contains supplementary material, which is available to authorized users.

Maria Gavriatopoulou

mariagabria@gmail.com

Extended author information available on the last page of the article hematopoietic cell transplantation, as well as novel therapies including proteasome inhibitors, immunomodulatory agents, monoclonal antibodies, and histone deacetylase inhibitors, have improved response rates and survival of patients over the past two decades [2-7]. These drugs have novel mechanisms of action compared with classical chemotherapies, and therefore unique toxicity profiles that require experience and supportive care strategies to manage them effectively. The toxicities of current antimyeloma therapies can be broadly classified as hematologic, gastrointestinal, neurological, cardiac, hepatic, immunologic, and teratogenic [8]. Knowledge of the optimal dose levels, routes of administration, and refinement of chemical structures have improved the effectiveness of these agents and treatment experience for patients over time [9-12].

Selinexor is an oral selective inhibitor of exportin 1 (XPO1) that has recently been approved by the US Food 
and Drug Administration for the treatment of patients with refractory MM [13]. In most cancer cells, including MM, XPO1 is overexpressed and associated with poor prognosis $[14,15]$. Selinexor reversibly binds to a critical cysteine residue (cys-528) in the nuclear export sequence-binding groove of XPO1 and prevents the transport of over 200 cargo proteins from the nucleus to the cytoplasm [16]. Inhibition of XPO1 retains tumor suppressor proteins in the nucleus leading to their activation, and prevents the nuclear export of mRNAs coding for oncoproteins, such as Myc, Bcl-6, and cyclin D1 [17]. These effects are lethal to cancer cells at concentrations that are not toxic to normal cells [18].

To date, selinexor has been administered to over 3000 patients with hematologic and solid cancers in clinical trials as a single agent or in combination with other therapies. The side effect profile of selinexor is well-established and predictable. The most common adverse events (AEs) are gastrointestinal (nausea, vomiting, and diarrhea), constitutional (fatigue, decreased appetite), hematologic (thrombocytopenia and neutropenia), and biochemical (hyponatremia) $[19,20]$. These AEs are largely dose and schedule dependent, reversible, and there is no evidence of major organ or cumulative toxicities after long-term treatment. AEs can be prevented or mitigated with prophylactic measures, and vigilant monitoring and management. Early incorporation of supportive care is key to address these side effects and maintain the patient's quality of life [19, 20].

Here, we describe a pooled analysis of patients with MM treated with selinexor, alone or in combinations, across four clinical trials. The objective of this analysis was to explore the effectiveness of supportive care agents and better understand the kinetics of the $\mathrm{AE}$ onset and resolution with and without intervention.

\section{Methods}

\section{Integrated analysis}

This was a retrospective, pooled analysis of 437 patients with MM from the phase 1 (NCT01607892) $(N=81)$, STORM (NCT02336815) $(N=202)$, STOMP (NCT02343042) $(N=$ $117)$, and BOSTON (NCT03110562) $(N=37)$ trials. Inclusion and exclusion criteria for each trial are listed in Supplementary Table 1 . The study protocols were approved by the institutional review board or an independent ethics committee at each participating center and were in accordance with the Declaration of Helsinki and the International Conference on Harmonization-Good Clinical Practice. All patients provided written informed consent prior to enrollment. Treatment-emergent adverse events (TEAE) were defined as any $\mathrm{AE}$ that developed, worsened, or became serious during the treatment period (time from first dose of selinexor (C1D1) to last dose of selinexor plus an additional 30 days of followup) regardless of causality. AEs were graded by the treating physician according to the Common Terminology Criteria for Adverse Events (CTCAE) version 4.03. An AE was considered resolved/resolving if there was an improvement in severity to a lower grade or recovery of an $\mathrm{AE}$ to normal without sequelae.

\section{Results}

\section{Demographics}

Baseline characteristics of all patients included in the analysis are outlined in Table 1. Patients had a median age of 64 years (range: $34-85$ ), had a median time since initial MM diagnosis of 5 years (range: $<1-35$ ), and were heavily pretreated ( $69 \% \geq 5$ prior lines of therapy). Of note, patients had a median of 13 (range: 1-62) distinct abnormalities in baseline clinical and laboratory tests at C1D1 of a selinexor trial with $43 \%$ having thrombocytopenia, $38 \%$ fatigue/ asthenia, $27 \%$ neutropenia, and $14 \%$ diarrhea.

Sixty-seven percent of the patients received selinexor on a twice-weekly schedule, $27 \%$ on a once-weekly schedule, and $6 \%$ on another schedule (primarily during the doseescalation phase of trials). The average starting dose received was $61-80 \mathrm{mg} /$ dose for $51 \%$ of patients, $81-100$ $\mathrm{mg} / \mathrm{dose}$ for $25 \%$ of patients, $>100 \mathrm{mg} / \mathrm{dose}$ for $3 \%$ of patients, and $<60 \mathrm{mg} /$ dose for $21 \%$ of patients. The median weekly dose for all patients was $100 \mathrm{mg}$ (range: 7-248 mg).

\section{Thrombocytopenia}

Decreased platelet count in patients with MM was the most common hematologic $\mathrm{AE}$ associated with selinexor treatment. Among the 437 patients in this analysis, $66 \%$ of patients developed thrombocytopenia of any grade $\left(<150,000\right.$ platelets $\left./ \mathrm{mm}^{3}\right), 22 \%$ developed grade 3 thrombocytopenia $\left(<50,000-25,000\right.$ platelets $\left./ \mathrm{mm}^{3}\right)$, and $32 \%$ developed grade 4 thrombocytopenia $(<25,000$ platelets/ $\mathrm{mm}^{3}$ ) while on study (Supplementary Table 2).

Platelet decrease was evident within the first 7 days of treatment and reached a nadir between 28 and 42 days in the absence of intervention (e.g., platelet transfusion, thrombopoietin (TPO) receptor agonist (TPO-RA), and/or dose reduction/interruption) Fig. 1a, b. Patients had a median baseline platelet count of 161,000 (quartile Q1 104,000, Q3 $214,000)$ and a median decrease of 85,000 platelets $/ \mathrm{mm}^{3}$ (Q1 45,000, Q3 141,000) from the start of selinexor through days 28-42. Importantly, for patients who continued therapy, there was no subsequent decrease in platelets from days 42 to 154 of treatment. The median time to onset of the first recorded thrombocytopenia was 22 days (Q1 12, Q3 
Table 1 Characteristics of patients at screening.

\begin{tabular}{|c|c|}
\hline Characteristic & $\begin{array}{l}\text { All patients } \\
N=437\end{array}$ \\
\hline \multicolumn{2}{|l|}{ Age (years) } \\
\hline Median (range) & $64(34-85)$ \\
\hline$\leq 65$ & $229(52 \%)$ \\
\hline $65-74$ & $160(37 \%)$ \\
\hline$\geq 75$ & $48(11 \%)$ \\
\hline \multicolumn{2}{|l|}{ Sex } \\
\hline Male & $236(54 \%)$ \\
\hline Female & $201(46 \%)$ \\
\hline \multicolumn{2}{|l|}{ Race } \\
\hline White & $341(78 \%)$ \\
\hline Black or African American & $55(13 \%)$ \\
\hline Asian & $10(2 \%)$ \\
\hline Other/unknown & $31(7 \%)$ \\
\hline \multicolumn{2}{|l|}{ ECOG performance status at screening } \\
\hline 0 & $108(25 \%)$ \\
\hline 1 & $280(64 \%)$ \\
\hline 2 & $38(9 \%)$ \\
\hline 3 & $1(<1 \%)$ \\
\hline Unknown & $10(2 \%)$ \\
\hline \multicolumn{2}{|l|}{ ISS disease stage at initial diagnosis } \\
\hline I & $95(22 \%)$ \\
\hline II & $87(20 \%)$ \\
\hline III & $127(29 \%)$ \\
\hline IV & $2(<1 \%)$ \\
\hline Unknown/missing & $126(29 \%)$ \\
\hline \multicolumn{2}{|l|}{ Median time since initial diagnosis of multiple myeloma } \\
\hline Years (range) & $5(<1-35)$ \\
\hline Number of prior therapeutic regimens & $6(0-18)$ \\
\hline 0 & $2(<1 \%)$ \\
\hline 1 & $42(10 \%)$ \\
\hline 2 & $27(6 \%)$ \\
\hline 3 & $26(6 \%)$ \\
\hline 4 & $37(8 \%)$ \\
\hline 5 & $56(13 \%)$ \\
\hline$\geq 6$ & $247(57 \%)$ \\
\hline \multicolumn{2}{|l|}{ Selinexor starting dose and treatment regimen } \\
\hline Selinexor $(80 \mathrm{mg})+$ dexamethasone $(20 \mathrm{mg})^{\mathrm{a}}$ & $214(49 \%)$ \\
\hline KCP-330-001 & $12(6 \%)^{\mathrm{b}}$ \\
\hline KCP-330-012 Part A & $79(37 \%)^{\mathrm{b}}$ \\
\hline KCP-330-012 Part B & $123(57 \%)^{\mathrm{b}}$ \\
\hline Selinexor $(<$ or $>80 \mathrm{mg}) \pm$ dexamethasone $(20 \mathrm{mg})$ & $70(16 \%)$ \\
\hline Selinexor/bortezomib/dexamethasone ${ }^{c}$ & $78(18 \%)$ \\
\hline Selinexor/lenalidomide/dexamethasone ${ }^{\mathrm{d}}$ & $24(5 \%)$ \\
\hline Selinexor/pomalidomide/dexamethasone $e^{\mathrm{e}}$ & $33(8 \%)$ \\
\hline Selinexor/daratumumab/dexamethasone ${ }^{f}$ & $18(4 \%)$ \\
\hline \multicolumn{2}{|l|}{ Abnormalities in baseline clinical and laboratory tests } \\
\hline Median (range) & $13(1-62)$ \\
\hline \multicolumn{2}{|l|}{ Nausea at screening } \\
\hline None & $386(88 \%)$ \\
\hline Grade 1 & $31(7 \%)$ \\
\hline Grade 2 & $4(<1 \%)$ \\
\hline Ongoing with unknown grade & $16(4 \%)$ \\
\hline \multicolumn{2}{|l|}{ Diarrhea at screening } \\
\hline None & $378(86 \%)$ \\
\hline Grade 1 & $36(8 \%)$ \\
\hline
\end{tabular}

Table 1 (continued)

\begin{tabular}{|c|c|}
\hline Characteristic & $\begin{array}{l}\text { All patients } \\
N=437\end{array}$ \\
\hline Grade 2 & $6(1 \%)$ \\
\hline Ongoing with unknown grade & $17(4 \%)$ \\
\hline \multicolumn{2}{|l|}{ Fatigue or asthenia at screening } \\
\hline None & $273(62 \%)$ \\
\hline Grade 1 & $95(22 \%)$ \\
\hline Grade 2 & $18(4 \%)$ \\
\hline Ongoing with unknown grade & $51(12 \%)$ \\
\hline \multicolumn{2}{|l|}{ Decreased appetite at screening } \\
\hline None & $411(94 \%)$ \\
\hline Grade 1 & $16(4 \%)$ \\
\hline Grade 2 & $4(<1 \%)$ \\
\hline Ongoing with unknown grade & $6(1 \%)$ \\
\hline \multicolumn{2}{|l|}{ Platelet count at screening } \\
\hline Above lower limit of normal & $249(57 \%)$ \\
\hline$<150,000-75,000 / \mathrm{mm}^{3}$ & $117(27 \%)$ \\
\hline$<75,000-50,000 / \mathrm{mm}^{3}$ & $39(9 \%)$ \\
\hline$<50,000-25,000 / \mathrm{mm}^{3}$ & $24(6 \%)$ \\
\hline$<25,000 / \mathrm{mm}^{3}$ & $6(1 \%)$ \\
\hline \multicolumn{2}{|l|}{ Neutrophil count at screening } \\
\hline Above lower limit of normal & $315(72 \%)$ \\
\hline$<2000-1500 / \mathrm{mm}^{3}$ & $64(15 \%)$ \\
\hline$<1500-1000 / \mathrm{mm}^{3}$ & $40(9 \%)$ \\
\hline$<1000-500 / \mathrm{mm}^{3}$ & $10(2 \%)$ \\
\hline$<500 / \mathrm{mm}^{3}$ & $2(<1 \%)$ \\
\hline \multicolumn{2}{|l|}{ Hemoglobin at screening } \\
\hline Above lower limit of normal & $46(11 \%)$ \\
\hline$<$ Lower limit of normal $-10.0 \mathrm{~g} / \mathrm{dL}$ & $182(42 \%)$ \\
\hline$<10.0-8.0 \mathrm{~g} / \mathrm{dL}$ & $174(40 \%)$ \\
\hline$<8.0 \mathrm{~g} / \mathrm{dL}$ & $34(8 \%)$ \\
\hline \multicolumn{2}{|l|}{ Plasma sodium at screening } \\
\hline Above lower limit of normal & $411(94 \%)$ \\
\hline Grade 1 & $18(4 \%)$ \\
\hline Grade 2 & $1(<1 \%)$ \\
\hline Grade 3 & $4(<1 \%)$ \\
\hline Ongoing with unknown grade & $3(<1 \%)$ \\
\hline \multicolumn{2}{|l|}{ Concomitant medications at screening } \\
\hline Median (range) & $7(0-21)$ \\
\hline
\end{tabular}

ECOG Eastern Cooperative Oncology Group, ISS International Staging System.

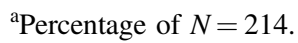

${ }^{\mathrm{b}}$ Selinexor (80 mg twice-weekly)/dexamethasone (20 mg twiceweekly).

${ }^{\mathrm{c}}$ Selinexor (60 or $80 \mathrm{mg}$ twice-weekly, or 80 or $100 \mathrm{mg}$ once-weekly)/ bortezomib $\left(1.3 \mathrm{mg} / \mathrm{m}^{2}\right.$ once-weekly or twice-weekly/dexamethasone (20 mg twice-weekly or $40 \mathrm{mg}$ once-weekly).

${ }^{\mathrm{d}}$ Selinexor (60 or $80 \mathrm{mg}$ twice-weekly, or 80 or $100 \mathrm{mg}$ once-weekly)/ lenalidomide ( $25 \mathrm{mg}$ once-daily)/dexamethasone ( $20 \mathrm{mg}$ twice-weekly or $40 \mathrm{mg}$ once-weekly).

${ }^{\mathrm{e}}$ Selinexor (60 or $80 \mathrm{mg}$ twice-weekly, or 60,80 or $100 \mathrm{mg}$ onceweekly)/pomalidomide $(2,3$, or $4 \mathrm{mg}$ once-daily)/dexamethasone ( 20 $\mathrm{mg}$ twice-weekly or $40 \mathrm{mg}$ once-weekly).

${ }^{\mathrm{f}}$ Selinexor (60 mg twice-weekly or $100 \mathrm{mg}$ once-weekly/daratumumab (intravenous, $16 \mathrm{mg} / \mathrm{kg}$ once-weekly)/dexamethasone $(20 \mathrm{mg}$ twiceweekly or $40 \mathrm{mg}$ once-weekly). 


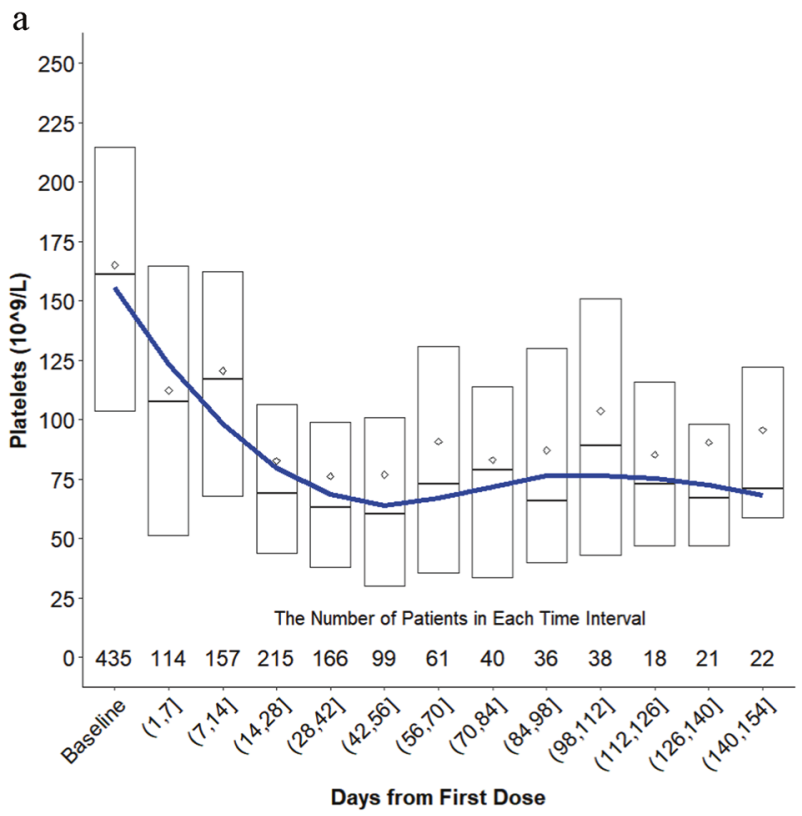

b

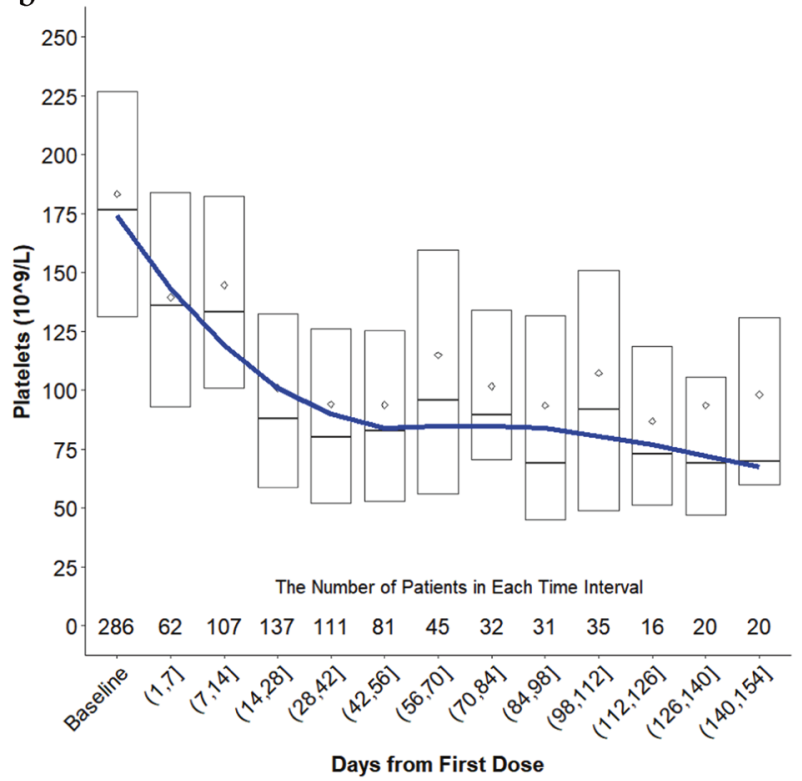

Fig. 1 Platelet change in patients during selinexor treatment. a Change in platelets from baseline (cycle 1 day 1 of treatment with selinexor or a selinexor-containing regimen) through day 154 for all patients with MM. Patients were included in the graph up until the point in time when they received an intervention for thrombocytopenia (platelet transfusion, TPO receptor agonist, dose reduction, and dose interruption) and were then subsequently removed from the analysis. b Change in platelets for all patients who did not receive a platelet transfusion or TPO receptor agonist while on a selinexor trial. Patients who received a dose reduction or interruption were included in the analysis up until the point in time where they had an intervention and were subsequently removed from the analysis.

29 days) and median time to onset of grade $\geq 3$ thrombocytopenia was 29 days (Q1 16, Q3 40 days). Severe bleeding (grade $\geq 3$ ) with corresponding grade $\geq 3$ thrombocytopenia was uncommon $(<3 \%)$. For patients who did not have an intervention for thrombocytopenia, 68 (44\%) of the AEs resolved or were resolving with the median duration of the AE being 52 days (Q1 24, Q3 134).

There were four strategies used to manage thrombocytopenia: platelet transfusions, TPO-RAs, dose reductions, and dose interruptions (Supplementary Fig. 1a-d). Platelet transfusions were primarily instituted for grade 4 thrombocytopenia (median 23,000 platelets $/ \mathrm{mm}^{3}$ at time of platelet transfusion; Q1 17 000, Q3 42 000). In total, 119 (27\%) patients had a platelet transfusion while on study. Eighty two (77\%) of the 107 patients that received a platelet transfusion and had a subsequent assessment showed resolution of thrombocytopenia in a median time of 8 days (Q1 3, Q3 16 days).

In preclinical models, TPO-RAs are effective at stimulating platelet production [21-23]. The clinical trials permitted use of the TPO-RAs romiplostim or eltrombopag to manage selinexor-induced thrombocytopenia, and 48 (11\%) patients received one of these agents. The median starting doses of eltrombopag and romiplostim were $50 \mathrm{mg}(\mathrm{Q} 150$, Q3 $50 \mathrm{mg}$ ) and $1 \mu \mathrm{g} / \mathrm{kg}(\mathrm{Q} 11$, Q3 $2 \mu \mathrm{g} / \mathrm{kg}$ ), respectively. The dose of romiplostim was incrementally increased each subsequent week up to $10 \mu \mathrm{g} / \mathrm{kg}$ if platelet counts were not restored. In general, higher doses of romiplostim or eltrombopag ( $\geq 5 \mu \mathrm{g} / \mathrm{kg}$ and $\geq 100 \mathrm{mg}$, respectively) were associated with a greater increase in platelet counts than lower doses. In addition, TPO-RAs were more effective with a once-weekly dosing schedule of selinexor compared with twice-weekly. The median time to first use of any TPO-RA was 36 days from baseline (Q1 29, Q3 45 days). The first evidence of thrombopoiesis for most patients occurred between 14 and 28 days and platelets steadily increased. Of the 48 patients who had a TEAE of thrombocytopenia and received a TPO-RA, 67\% had their AE resolved or resolving in a median time of 14 days (Q1 7, Q3 23 days).

Overall, both dose reduction and interruption were effective at mitigating thrombocytopenia; however, platelet loss persisted for an additional 7-14 days after dose reduction or interruption $\left(\sim 22,000\right.$ platelets $/ \mathrm{mm}^{3}$ to nadir). The median time to first dose reduction and interruption for thrombocytopenia occurred at 36 days (range: $8-722$ days) and 32 days (range: 4-1021 days), respectively. Dose reductions for thrombocytopenia occurred in 141 (32\%) patients with the most common dose and schedule change from selinexor $80 \mathrm{mg}$ twice-weekly to $100 \mathrm{mg}$ once-weekly.

\section{Neutropenia}

Decreased neutrophil count occurred in 163 (37\%) patients $\left(<2000\right.$ neutrophils $\left./ \mathrm{mm}^{3}\right)$ with $85(20 \%)$ patients having grade 3 neutropenia $\left(<1000-500\right.$ neutrophils $\left./ \mathrm{mm}^{3}\right)$ and 38 
(9\%) having grade 4 neutropenia $\left(<500\right.$ neutrophils $\left./ \mathrm{mm}^{3}\right)$ while on study (Supplementary Table 2). Febrile neutropenia occurred in $19(4 \%)$ of the 437 patients in this analysis. Severe (grade $\geq 3$ ) infections in the context of grade $\geq 3$ neutropenia occurred in 23 patients $(19 \%)$ and included Clostridium difficile infection (1 patient), bacteremia (2 patients), upper respiratory tract infection (2 patients), influenza (3 patients), sepsis (3 patients), pneumonia, or lung infection (12 patients).

Neutropenia began within the first 7 days of treatment and reached a nadir between 28 and 42 days in the absence of intervention (granulocyte colony stimulating factor (GCSF) or dose reduction/interruption) (Fig. 2a). The median time to onset of any grade of neutropenia was 21 days (Q1 8, Q3 43 days) and was 26 days (Q1 15, Q3 50 days) for grade $\geq 3$. The median duration of neutropenia among all patients, regardless of intervention, was 10 days (Q1 7, Q3 18). Patients had a median decrease of 530 neutrophils $/ \mathrm{mm}^{3}$ (Q1 0, Q3 1700 neutrophils $/ \mathrm{mm}^{3}$ ) from the start of selinexor through days $28-42$. There was no substantial decrease in neutrophil count between days 42 and 154 of treatment.

The G-CSF agents filgrastim and pegfilgrastim were used in $92(75 \%)$ patients with grade $\geq 3$ neutropenia and were most commonly administered as a single injection (median 1 dose; Q1 1, Q3 3 doses) at $300 \mu \mathrm{g}$ or $6 \mathrm{mg}$ subcutaneously, respectively. Both filgrastim and pegfilgrastim were effective at rapidly (within 7 days) and durably raising neutrophil counts (Fig. 2b). These supportive care agents were instituted at a median of 800 neutrophils $/ \mathrm{mm}^{3}$ and increased neutrophil levels to 1580 neutrophils $/ \mathrm{mm}^{3}$ (Q1 1000, Q3 3400) within the first week. Neutrophil levels continued to improve over the subsequent weeks. Neutropenia resolved or was resolving in $90 \%$ of patients that received filgrastim or pegfilgrastim versus $64 \%$ of patients that did not. Patients who received a G-CSF had a median time to resolution of neutropenia of 8 days (Q1 5, Q3 15).

\section{Nausea/vomiting}

The most common AE observed with selinexor treatment is nausea, which occurred in $68 \%$ of patients with grade 3 nausea in $6 \%$ of patients; vomiting occurred in $37 \%$ of patients. The median time to onset of any grade nausea/ vomiting (N/V) was 3 days (Q1 2, Q3 8) and median time to onset of grade $3 \mathrm{~N} / \mathrm{V}$ was 16 days (Q1 3, Q3 31 days). For all patients, the onset of N/V was highest within the first cycle of treatment (70\%) and dropped substantially for each subsequent cycle. Fifty-eight percent of patients with N/V had corresponding fatigue, $51 \%$ decreased appetite, $34 \%$ weight loss, $27 \%$ hyponatremia, and $17 \%$ dehydration.

The average duration of N/V was 22 days (Q1 6, Q3 51) for patients who did not receive supportive care within 5 days of onset and was 13 days (Q1 5, Q3 34 days) for
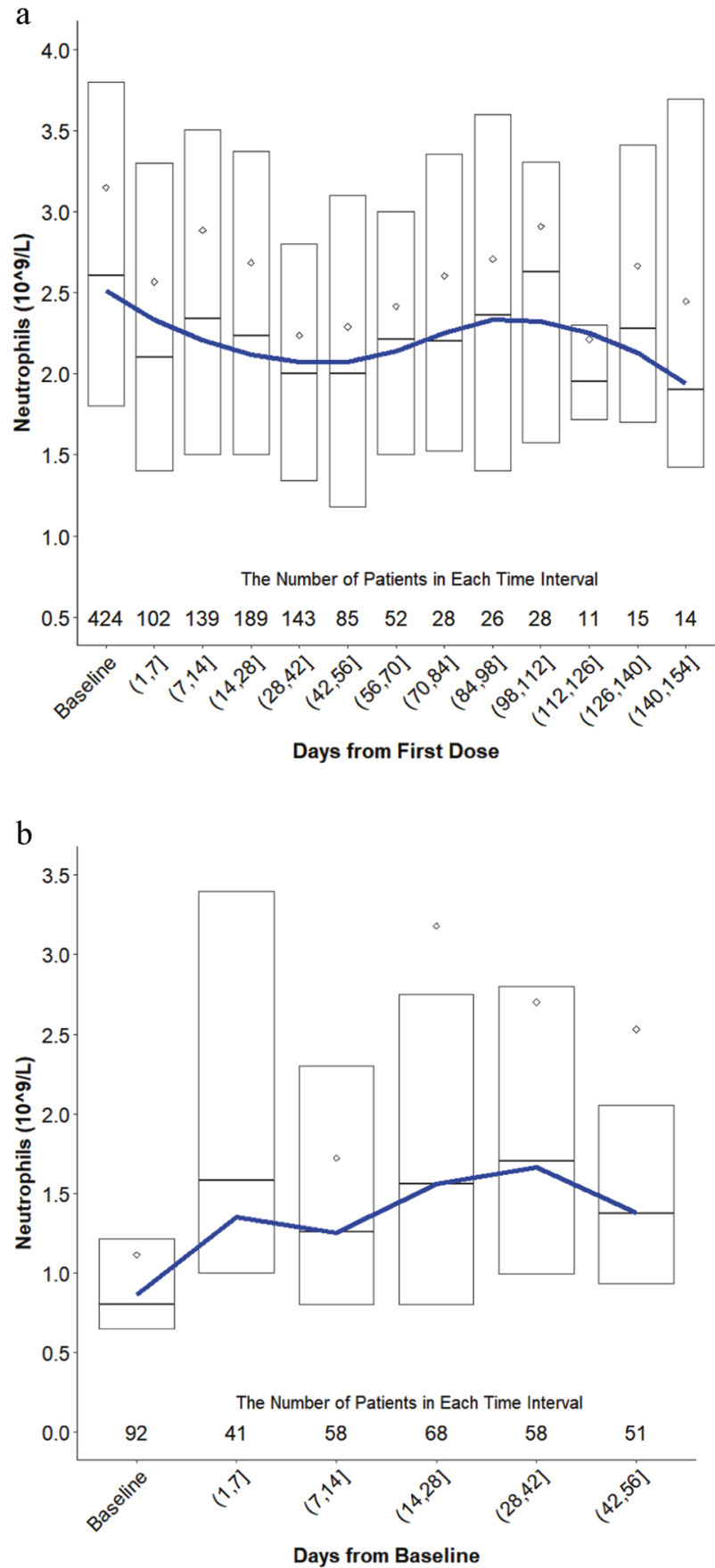

Fig. 2 Neutrophil change in patients during selinexor treatment and after a G-CSF. a Change in neutrophils from baseline (C1D1 of treatment with selinexor or a selinexor-containing regimen) through day 154 for all patients with MM. Patients were included in the graph up until the point in time when they received an intervention for neutropenia (filgrastim/pegfilgrastim, dose reduction, and dose interruption) and were then subsequently removed from the analysis. b Change in neutrophils for all patients who received filgrastim or pegfilgrastim.

patients who did receive such care. A full summary of the supportive care agents that were used to manage N/V can be found in Table 2. Most patients received a mandatory 
5-HT3 antagonist on the day of and day after dosing. Additional agents improved the frequency of resolution and substantially reduced the median duration of N/V. In particular, neurokinin 1 (NK1) RAs (rolapitant [180 mg], aprepitant $[80 \mathrm{mg}$ ], or fosaprepitant [ $150 \mathrm{mg}]$ prior to study drug administration and as needed), benzodiazepenes (lorazepam $0.5-1 \mathrm{mg}$ daily as needed), and cannabinoid-RAs (dronabinol 2.5-5 mg twice-daily as needed) were effective at resolving $\mathrm{N} / \mathrm{V}$ and/or substantially reducing the duration of this AE.

Consistent with a previous report, prophylactic olanzapine (2.5-5 mg orally in the evening) or megestrol (400 mg daily) for N/V (and decreased appetite discussed below) were used in 39 (9\%) patients [24]. Incidence and severity of N/V were numerically lower in patients who received prophylactic olanzapine and megestrol (any grade, 56\%; grade 3,3\%), compared with patients who did not (any grade, $70 \%$; grade $3,7 \%$ ).

\section{Diarrhea}

There were 177 (41\%) patients who reported diarrhea while taking selinexor with a median duration of 4 days (Q1 3, Q3 15 days). Twenty-two (5\%) of the patients had grade 3 diarrhea with no grade $\geq 4$. The median time to onset for the first event of any grade diarrhea was 13 days (Q1 5, Q3 36 days). Seventy-two percent of reported cases of diarrhea were resolved or resolving in a median time of 7 days $(\mathrm{Q} 1$ 3, Q3 24 days). In general, diarrhea was associated with fatigue $(45 \%)$, decreased appetite (40\%), decreased body weight (24\%), and hyponatremia (21\%). Grade 3 diarrhea was most commonly associated with grade $\geq 3$ hyponatremia $(18 \%)$ and fatigue $(9 \%)$. Diarrhea resolved or was resolving in $87 \%$ of patients who received either loperamide or bismuth subsalicylate.

\section{Fatigue}

Fatigue is a common $\mathrm{AE}$ among patients with heavily pretreated $\mathrm{MM}$ and was one of the most frequently observed TEAEs in selinexor trials. Importantly, 38\% of the 437 patients analyzed in this study had ongoing fatigue during the screening period prior to selinexor treatment. Overall, $63 \%$ of patients reported any grade of fatigue while on a selinexor trial, with a median time-toonset of 7 day (Q1 3, Q3 20 days); 16\% had grade 3 fatigue with a median time to onset of 22 days (Q1 8, Q3 $50)$. In the majority $(70 \%)$ of patients, fatigue did not resolve, but for those in whom fatigue resolved or was resolving, the median duration of this $\mathrm{AE}$ was 29 days (Q1 11, Q3 67 days). Fatigue was most commonly associated with decreased appetite (54\%), anemia (48\%), decreased body weight (34\%), and dehydration (15\%).

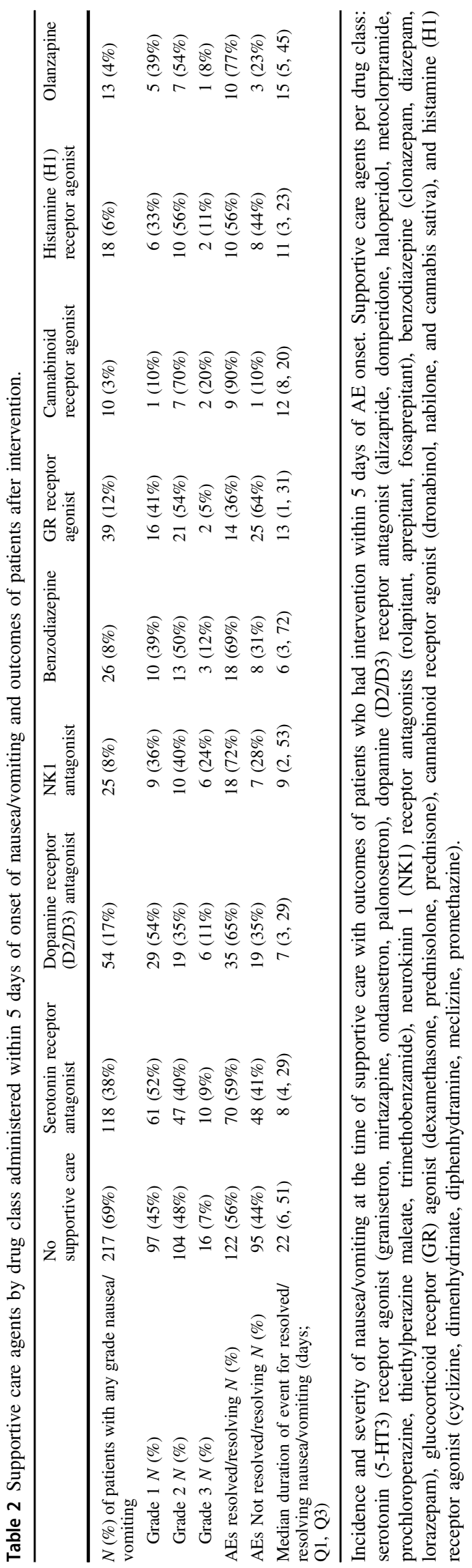


Table 3 Supportive care agents by drug class administered within 5 days of onset of decreased appetite and outcomes of patients after intervention.

\begin{tabular}{|c|c|c|c|c|c|}
\hline & $\begin{array}{l}\text { No } \\
\text { supportive care }\end{array}$ & $\begin{array}{l}\text { Megestrol } \\
\text { acetate }\end{array}$ & $\begin{array}{l}\text { Cannabinoid } \\
\text { receptor agonist }\end{array}$ & $\begin{array}{l}\text { Glucocorticoid } \\
\text { receptor agonist }\end{array}$ & $\begin{array}{l}\text { Mirtazapine or } \\
\text { olanzapine }\end{array}$ \\
\hline $\begin{array}{l}N(\%) \text { of patients with any grade } \\
\text { decreased appetite }\end{array}$ & $197(53 \%)$ & $22(10 \%)$ & $13(6 \%)$ & $26(11 \%)$ & $12(5 \%)$ \\
\hline Grade $1 N(\%)$ & $87(44 \%)$ & $6(27 \%)$ & $3(23 \%)$ & $10(39 \%)$ & $4(33 \%)$ \\
\hline Grade $2 N(\%)$ & $96(49 \%)$ & $15(68 \%)$ & $10(77 \%)$ & $11(42 \%)$ & $7(58 \%)$ \\
\hline Grade $3 N(\%)$ & $14(7 \%)$ & $1(5 \%)$ & - & $5(19 \%)$ & $1(8 \%)$ \\
\hline AEs resolved/resolving $N(\%)$ & $87(44 \%)$ & $14(64 \%)$ & $10(77 \%)$ & $8(31 \%)$ & $6(50 \%)$ \\
\hline AEs not resolved/resolving $N(\%)$ & $110(56 \%)$ & $8(36 \%)$ & $3(23 \%)$ & $18(69 \%)$ & $6(50 \%)$ \\
\hline $\begin{array}{l}\text { Median duration of event for resolved } \\
\text { decreased appetite (days; Q1, Q3) }\end{array}$ & $36(15,109)$ & $21(8,29)$ & $41(19,54)$ & $14(3,33)$ & $8(6,25)$ \\
\hline
\end{tabular}

Incidence and severity of decreased appetite at the time of supportive care with outcomes of patients who had intervention within 5 days of $\mathrm{AE}$ onset. Supportive care agents per drug class: cannabinoid receptor agonist (dronabinol, nabilone, cannabis sativa, glucocorticoid receptor (GR) agonist (dexamethasone, prednisolone, prednisone).

Methylphenidate was used either prophylactically or on study in 12 patients treated with selinexor. Of these 12 patients, three were treated upon emergence of fatigue with daily doses of methylphenidate $\leq 5 \mathrm{mg}$, and none saw a resolution of or improvement in fatigue (Supplementary Table 3). The remaining nine patients were treated at doses $\geq 10 \mathrm{mg}$ of methylphenidate. Three patients were treated prophylactically, and none reported fatigue while on study. The other six received methylphenidate as needed, with five of the six patients having a resolution of their fatigue. The median time to resolution of fatigue with methylphenidate was 6 days (Q1 4, Q3 8 days).

For fatigue that was attributed to insomnia, mirtazapine or olanzapine were used in 13 patients. Fatigue resolved or was resolving in $8(62 \%)$ of these patients with a median duration of 10 days (Q1 6, Q3 42 days).

\section{Decreased appetite/weight loss}

Decreased appetite occurred in $53 \%$ of patients, with grade 3 in 7\%. The median time to onset of decreased appetite was 8 days (Q1 3, Q3 22 days), and the median time to onset for grade $\geq 3$ was 35 days (Q1 24, Q3 44 days). The primary AEs that were associated with decreased appetite were fatigue $(66 \%)$, nausea (59\%), weight loss $(47 \%)$, diarrhea $(41 \%)$, vomiting $(33 \%)$, dehydration $(17 \%)$, and dysgeusia (17\%). In $56 \%$ of patients, decreased appetite did not resolve without intervention. For the $46 \%$ with decreased appetite that resolved or resolving in the absence of intervention, the median duration was 36 days (Q1 15, Q3 109 days).

The primary supportive care measures to manage decreased appetite and accompanied weight loss were the use of megestrol (10\%), cannabinoids (6\%) (cannabis sativa, dronabinol), or olanzapine or mirtazapine $(5 \%)$ (Table 3). Typical doses were $400 \mathrm{mg}$ daily megestrol acetate, $2.5 \mathrm{mg}$ twice-daily dronabinol, or $5 \mathrm{mg}$ nightly olanzapine. For patients who received supportive care within 5 days of onset of decreased appetite, the frequency of $\mathrm{AE}$ resolution was $64 \%$ with megestrol, $77 \%$ with cannabinoids, and $50 \%$ with olanzapine or mirtazapine. The average duration of decreased appetite was 21 days (Q1 8, Q3 29 days) with megestrol, 41 days (Q1 19, Q3 54 days) with cannabinoids, and 8 days (Q1 6, Q3 25 days) with olanzapine or mirtazapine. Of the 39 patients who received prophylactic olanzapine and megestrol, $31 \%$ had decreased appetite versus $50 \%$ who did not receive such prophylaxis.

\section{Hyponatremia}

Hyponatremia (sodium $<135 \mathrm{mmol} / \mathrm{L}$ ) was observed in 138 (32\%) of the 437 patients while on study and 83 (19\%) patients had grade $\geq 3$ hyponatremia ( 120 to $<130$ $\mathrm{mmol} / \mathrm{L}$ ); there is no grade 2 hyponatremia in CTCAE v4.03. The mechanism behind treatment-emergent hyponatremia is not completely understood and the etiology is multifactorial. Importantly, a majority of grade $\geq 3$ hyponatremia was asymptomatic, with $<5 \%$ of the cases associated with grade $\geq 3$ dizziness, confusion, altered mental status, or delirium.

The median time to onset of any grade of hyponatremia was 8 days (Q1 5, Q3 18 days) with the median time to onset of grade $\geq 3$ hyponatremia being 10 days (Q1 6, Q3 24 days). The primary supportive care measure for managing hyponatremia was sodium chloride tablets. Of the 60 patients who received this supportive care, $83 \%$ had resolution of hyponatremia versus $65 \%$ that did not receive sodium chloride. Furthermore, the median time to resolution of hyponatremia was 7 days (Q1 3, Q3 13) for patients who received sodium chloride and 12 days (Q1 6, Q3 20 days) for patients who did not. 


\section{Discussion}

This is the first paper to describe a set of pooled AE data from patients with MM treated with selinexor as a single agent or in combination. We show the kinetics of the most common selinexor TEAEs and provide practice-based prophylactic and supportive care strategies to address them (Table 4). The most common side effects were reversible and consistent with previous reports and included thrombocytopenia, neutropenia, nausea, vomiting, diarrhea, fatigue, decreased appetite, and hyponatremia [19, 25-27]. Most patients who were treated with selinexor required concomitant prophylactic and on study supportive care, dose modifications, and vigilant monitoring to prevent or avoid worsening of AEs. Clinical sequellae of cytopenias and low sodium were not commonly observed, and major organ (cardiac, hepatic, renal, neural) or cumulative toxicities were not typical.

The primary hematological AEs with selinexor treatment are thrombocytopenia and, to a lesser extent, neutropenia. Associated bleeding or febrile neutropenia were uncommon. Preclinical studies have demonstrated that selinexor prevents hematopoietic stem and progenitor cell maturation into megakaryocytes through inhibition of TPO signaling and accumulation of phosphorylated STAT3 in the nucleus, rather than direct cytotoxic effects on hematopoietic stem cells, megakaryocytes, or platelets [21]. When considering the patient population in this analysis, it is important to note that in addition to substantial marrow involvement by their myeloma, nearly all of the patients had received previous myelosuppressive treatments, and $43 \%$ and $28 \%$ of the patients had preexisting thrombocytopenia and neutropenia, respectively. Previous reports showed that platelet counts at baseline were predictive of developing high-grade thrombocytopenia, with nearly all patients with platelet counts $<75,000 / \mathrm{mm}^{3}$ at baseline having grade $\geq 3$ thrombocytopenia while on study [26]. Physicians should consider weekly monitoring of blood counts, particularly in patients with low baseline counts. The data show that platelet transfusions were effective at rapidly boosting platelet levels for patients with severe thrombocytopenia, and that romiplostim or eltrombopag, albeit off-label use of these drugs, could be used to increase platelet counts over $2-3$ weeks, often while continuing selinexor treatment. G-CSF was highly effective at rapidly normalizing low neutrophil counts.

Gastrointestinal AEs, in particular N/V, are the most common nonhematological side effects of selinexor treatment and require prophylactic supportive care and additional on study management to limit duration and severity. The median onset of N/V occurred at day 3, suggesting delayed emesis, which should be properly managed $[28,29]$. Previous studies have suggested that selinexorinduced N/V is likely mediated through the central nervous system, as selinexor crosses the blood brain barrier and has anticancer activity in the brain [30-32]. Prophylactic use of a 5-HT3 RAs (ondansetron or equivalent) in all patients, was mandated on selinexor trials after phase 1 to reduce $\mathrm{N} / \mathrm{V}$. The use of a second antiemetic, such as $2.5-10 \mathrm{mg}$ olanzapine in the evening and/or NK1-RAs should be strongly considered prior to the first day of therapy.

Table 4 Summary of recommended supportive care guidelines and patient management with selinexor treatment.

Patient management

Prophylaxis for Nausea and/or Vomiting ${ }^{\text {a }}$

Diarrhea

Hyponatremia

Thrombocytopenia ${ }^{\mathrm{b}}$

Neutropenia ${ }^{c}$

Fatigue $^{\mathrm{d}}$
Weekly monitoring of blood counts, serum sodium, and body weight for the first 8 weeks and as needed thereafter.

All patients should receive a 5-HT3 receptor antagonist (e.g., ondansetron, $8 \mathrm{mg}, \mathrm{PO}, 30 \mathrm{~min}$ prior to first dose of selinexor, with subsequent $8 \mathrm{mg}$ dose $8 \mathrm{~h}$ after the first dose of selinexor; then administered $8 \mathrm{mg}$ twice-daily (every $12 \mathrm{~h}$ with coverage for $24 \mathrm{~h}$ after the last dose of selinexor); continued use should be evaluated after the first 8 weeks. Patients at high-risk for nausea and/or vomiting, or decreased appetite should receive an additional agent such as olanzapine (5-10 mg, PO, once-daily in the evening) or NK1 receptor antagonist (e.g., rolapitant per label). If using aprepritant or fosapreptiant the dose of dexamethasone may need to be reduced. Olanzapine or NK1 receptor antagonists may be reduced or stopped after the first 8 weeks if the patient is tolerating selinexor treatment.

Loperamide (4 $\mathrm{mg}$ for the first dose, and $2 \mathrm{mg}$ thereafter, $\mathrm{PO}$, as needed) until diarrhea resolves.

Hydration status and serum sodium should be monitored, and saline/salt tablets employed as needed.

TPO receptor agonist for platelet counts below $25,000 / \mathrm{mm}^{3}$ (romiplostim, 5-10 mcg/kg, IV, once-weekly; or eltrombopag, $50 \mathrm{mg}$, PO, daily, until platelet counts recover to $\geq 50,000 / \mathrm{mm}^{3}$ ).

G-CSF for ANC below 500/ $\mathrm{mm}^{3}$ (filgrastim, SC or IV, $5 \mathrm{mcg} / \mathrm{kg}$ ), daily until neutrophil count resolves to $>1000$ / $\mathrm{mm}^{3}$; or pegfilgrastim, SC, $6 \mathrm{mg}$, weekly until neutrophil count resolves to $>1000 / \mathrm{mm}^{3}$ ).

Establish causative relationship between treatment regimen and fatigue (consider onset, pattern, duration, change over time, as well as the patient's disease status and self-assessment of causes of fatigue).

For grade 2 or 3 fatigue, methylphenidate $(10 \mathrm{mg}, \mathrm{PO}$, daily) as needed.

${ }^{\mathrm{a}}$ Follow NCCN and/or European Society for Medical Oncology (ESMO) guidelines for moderate emetogenic chemotherapies (MECs) and treat for both acute and delayed emesis.

${ }^{b}$ Recommendation is off-label use of romiplostim and eltrombopag.

${ }^{c}$ Follow 2019 NCCN guidelines: hematopoietic growth factors.

${ }^{\mathrm{d}}$ Consider 2019 NCCN guidelines for the treatment of cancer related fatigue. 
Additional antinausea medications may be required for patients who experience on study gastrointestinal AEs, and the data shown here suggest that NK1-RAs, benzodiazepines, and cannabinoid-RAs were effective at mitigating treatment-emergent N/V. As suggested by the association data, several AEs are linked to N/V, including decreased appetite, weight loss, hyponatremia, and fatigue, suggesting that management of N/V may limit the incidence of these AEs. Diarrhea was less common in the study population and was generally responsive to loperamide or bismuth subsalicylate.

The constitutional $\mathrm{AE}$ of fatigue requires early and vigilant management while patients are receiving selinexor treatment. Physicians should refer to the National Comprehensive Cancer Network (NCCN) Guidelines for the treatment of cancer related fatigue, as well as closely monitor for and correct underlying contributors to fatigue [33]. Methylphenidate at doses of $\geq 10 \mathrm{mg}$ in the morning proved to be the most effective supportive care strategy for attenuating the symptoms of fatigue, with 8 out of 9 patients never having fatigue or having it resolve with use.

Decreased appetite and weight loss can occur with selinexor treatment and were also the primary side effects observed preclinically in animal models [34]. Nutritional counseling, dose modifications, and appetite stimulants may prevent or improve weight loss. For patients with decreased appetite, megestrol, cannabinoids, and mirtazapine or olanzapine were effective at resolving anorexia and reducing its duration. Physicians should also monitor for corresponding N/V or dysgeusia, which can substantially impact a patient's desire to eat. Olanzapine $(2.5-10 \mathrm{mg})$ used in the evening and begun prior to the first dose of selinexor appears to address both $\mathrm{N} / \mathrm{V}$ and decreased appetite.

Hyponatremia is a common $\mathrm{AE}$ observed with selinexor treatment with the highest incidence occurring in MM trials. A majority ( $>95 \%)$ of the hyponatremia was asymptomatic, and generally correctable with sodium tablets or electrolytecontaining fluids. The causes of hyponatremia are not completely understood and are likely to be multifactorial. Furthermore, pseudohyponatremia in many cases could not be ruled out and can be caused by elevated paraprotein levels and/or hyperglycemia. Appropriate laboratory methods using direct potentiometry in a gas analyzer are required to correct for pseudohyponatremia.

There are several limitations of this analysis which should be considered when interpreting the results. Firstly, the data were pooled from four independent clinical trials that treated patients with MM at different stages of their disease, and $35 \%$ of the patients received combination therapies which could exacerbate AE prevelance and grade. Among these studies, there was a wide range of baseline characteristics, including: prior treatment history $(0-3$ prior lines; $22 \%$, and $\geq 4$ prior lines, $78 \%$ of patients), disease burden, frailty, ECOG performance status, as well as differences in the treatment schedule, dose, and regimen. Previous studies have demonstrated that $\mathrm{AE}$ burden increases with each line of therapy, age, and frailty of patients [35]. While the majority of the patients were heavily pretreated (median 6 prior lines of therapy), $22 \%$ of the population analyzed received $\leq 3$ prior antimyeloma regimens, which could impact onset, duration, and resolution of specific AEs. An additional limitation to this analysis is that patients may have received multiple interventions, at different times of $\mathrm{AE}$ onset, and controlling for these interventions and interpreting the effect in small subsets of patients was challenging. Furthermore, as knowledge of the AE profile of selinexor improved with clinical experience, prophylactic supportive care and early follow-up to mitigate $\mathrm{AE}$ severity became more common, resulting in nonstandardized or controlled AE prevention and management.

In conclusion, selinexor is a novel anticancer therapy that is being integrated in the therapeutic algorithm for patients with relapsed/refractory MM. The side effects of selinexor are unique amongst myeloma drugs, and are generally reversible with dose modification and supportive care. Clincial trials are ongoing to investigate selinexor in earlier lines of therapy and at lower doses in combination with approved agents, with the goal of maximizing efficacy and reducing side effects. Further studies examining AE mitigation strategies in a large, standardized patient population will provide further insight and help establish additional guidelines for the management of common AEs related to selinexor treatment.

Acknowledgements The authors thank the patients who participated in these trials and their families, the coinvestigators, nurses, and study coordinators at each of the sites.

\section{Compliance with ethical standards}

Conflict of interest MG: honoraria from Amgen, Karyopharm, Takeda, Genesis, Janssen-Cilag. AC: grant support, advisory board fees, research funding, and/or consulting fees from Takeda, Celgene, Novartis, Amgen, Janssen, BMS, Sanofi, Oncopeptides, Pharmacyclics, Seattle Genetics, Array BioPharma. DV: consulting, research funding, and/or advisory role Celgene, Amgen, Karyopharm, Teva, Janssen, Millennium, Acetylon, GlaxoSmithKline, Calithera Biosciences, Constellation. AJ: research funding, honoraria, consulting, and/or advisory role Karyopharm, Amgen, Abbvie, Celgene, Janssen, Takeda, Sanofi, SkylineDx. DD: research funding Takeda, Karyopharm. CH: grant or personal fees Janssen, Celgene, Karyopharm, Oncopeptides, Adaptive Biotechnologies. DS: speakers' bureau and/or funding: Celgene, Amgen, Janssen, Takeda, BMS. DW: consultancy fees and honoraria Amgen, Celgene, Janssen, Sanofi, Takeda. SL: advisor and/or shareholder Caelum Biosciences, Janssen, Bayer. CAH: fees/travel/advisory/and/or grant support Janssen, Celgene, Sanofi, Karyopharm, Glenmark. SJ: advisory or consulting fees Celgene, BMS, Janssen, Merck. RB: consulting, advisory role, and/or funding Celgene, Karyopharm, Signal Genetics, Takeda, Merck. AN: advisory 
board fees Amgen, GlaxoSmithKline, BMS, Celgene, Takeda, Janssen, Spectrum, Adaptive Biotechnologies. JR: consulting fees and speakers bureau Amgen, Celgene, Takeda, Janssen, Sanofi, Karyopharm, Oncopeptides, Adaptive Biotechnologies, and BMS. RA: honoraria, consulting, advisory role, and/or speaker's bureau Celgene, Amgen, Takeda, Janssen, BMS, Karyopharm. AY: consulting fees or grant support Adaptive Biotechnologies, Amgen, Karyopharm, Takeda, Janssen, Dexcel Pharma, BMS, Celgene. PM: honoraria Janssen, Celgene, Takeda, Amgen, and AbbVie. SL: advisory board fees Celgene, Takeda, Janssen, Novartis, BMS, and GlaxoSmithKline. ST: consulting/speaker's bureau/advisory board or grant support Celgene, Amgen, Janssen, Sanofi, Merck, Alnylam. KW: honoraria/consulting/advisory role/funding Amgen, BMS, Celgene, Janssen, Novartis, Onyx, Takeda, Sanofi. MM: grant support/fees Janssen, Sanofi, Jazz Celgene, BMS, Takeda Amgen, Roche. TU, KL, YC, LL, JS, SS, MK: employees/stockholders in Karyopharm. SS: Founder/ President/CSO Karyopharm. MK: CEO Karyopharm. MD: honorarium/consulting/advisory role/funding Amgen, Novartis, Celgene, Takeda, Genesis, Janssen, BMS. The remaining authors report no relevant conflicts of interest.

Publisher's note Springer Nature remains neutral with regard to jurisdictional claims in published maps and institutional affiliations.

Open Access This article is licensed under a Creative Commons Attribution 4.0 International License, which permits use, sharing, adaptation, distribution and reproduction in any medium or format, as long as you give appropriate credit to the original author(s) and the source, provide a link to the Creative Commons license, and indicate if changes were made. The images or other third party material in this article are included in the article's Creative Commons license, unless indicated otherwise in a credit line to the material. If material is not included in the article's Creative Commons license and your intended use is not permitted by statutory regulation or exceeds the permitted use, you will need to obtain permission directly from the copyright holder. To view a copy of this license, visit http://creativecommons. org/licenses/by/4.0/.

\section{References}

1. Cowan AJ, Allen C, Barac A, Basaleem H, Bensenor I, Curado MP, et al. Global burden of multiple myeloma: a systematic analysis for the global burden of disease study 2016. JAMA Oncol. 2018;4:1221-7.

2. San Miguel JF, Hungria VTM, Yoon S-S, Beksac M, Dimopoulos MA, Elghandour A, et al. Overall survival of patients with relapsed multiple myeloma treated with panobinostat or placebo plus bortezomib and dexamethasone (the PANORAMA 1 trial): a randomised, placebo-controlled, phase 3 trial. Lancet Haematol. 2016;3:e506-15.

3. Siegel DS, Martin T, Wang M, Vij R, Jakubowiak AJ, Lonial S, et al. A phase 2 study of single-agent carfilzomib (PX-171-003A1) in patients with relapsed and refractory multiple myeloma. Blood. 2012;120:2817-25.

4. Durie BGM, Hoering A, Abidi MH, Rajkumar SV, Epstein J, Kahanic SP, et al. Bortezomib with lenalidomide and dexamethasone versus lenalidomide and dexamethasone alone in patients with newly diagnosed myeloma without intent for immediate autologous stem-cell transplant (SWOG S0777): a randomised, open-label, phase 3 trial. Lancet. 2017;389:519-27.

5. Richardson PG, Sonneveld P, Schuster M, Irwin D, Stadtmauer E, Facon T, et al. Extended follow-up of a phase 3 trial in relapsed multiple myeloma: final time-to-event results of the APEX trial. Blood. 2007;110:3557-60.
6. Richardson P, Jagannath S, Hussein M, Berenson J, Singhal S, Irwin $\mathrm{D}$, et al. Safety and efficacy of single-agent lenalidomide in patients with relapsed and refractory multiple myeloma. Blood. 2009;114:772-8.

7. Dimopoulos MA, Moreau P, Palumbo A, Joshua D, Pour L, Hájek $\mathrm{R}$, et al. Carfilzomib and dexamethasone versus bortezomib and dexamethasone for patients with relapsed or refractory multiple myeloma (ENDEAVOR): a randomised, phase 3, open-label, multicentre study. Lancet Oncol. 2016;17:27-38.

8. Ludwig H, Delforge M, Facon T, Einsele H, Gay F, Moreau P, et al. Prevention and management of adverse events of novel agents in multiple myeloma: a consensus of the European Myeloma Network. Leukemia. 2018;32:1542-60.

9. Moreau P, Mateos M-V, Berenson JR, Weisel K, Lazzaro A, Song $\mathrm{K}$, et al. Once weekly versus twice weekly carfilzomib dosing in patients with relapsed and refractory multiple myeloma (A.R.R.O. W.): interim analysis results of a randomised, phase 3 study. Lancet Oncol. 2018;19:953-64.

10. Mu S-D, Ai L-S, Qin Y, Hu Y. Subcutaneous versus Intravenous Bortezomib Administration for Multiple Myeloma Patients: a Meta-analysis. Curr Med Sci. 2018;38:43-50.

11. Sehgal K, Das R, Zhang L, Verma R, Deng Y, Kocoglu M, et al. Clinical and pharmacodynamic analysis of pomalidomide dosing strategies in myeloma: impact of immune activation and cereblon targets. Blood. 2015;125:4042-51.

12. San Miguel JF, Hungria VTM, Yoon S-S, Beksac M, Dimopoulos MA, Elghandour A, et al. Panobinostat plus bortezomib and dexamethasone: impact of dose intensity and administration frequency on safety in the PANORAMA 1 trial. Br J Haematol. 2017;179:66-74.

13. XPOVIOTM (selinexor). Prescribing information. Reference ID: 4457635-US FDA

14. Schmidt J, Braggio E, Kortuem KM, Egan JB, Zhu YX, Xin CS, et al. Genome-wide studies in multiple myeloma identify XPO1/ CRM1 as a critical target validated using the selective nuclear export inhibitor KPT-276. Leukemia. 2013;27:2357-65.

15. Tai Y-T, Landesman Y, Acharya C, Calle Y, Zhong MY, Cea M, et al. CRM1 inhibition induces tumor cell cytotoxicity and impairs osteoclastogenesis in multiple myeloma: molecular mechanisms and therapeutic implications. Leukemia. 2014;28:155-65.

16. Fung HYJ, Chook YM. Atomic basis of CRM1-cargo recognition, release and inhibition. Semin Cancer Biol. 2014;27:52-61.

17. Gravina GL, Senapedis W, McCauley D, Baloglu E, Shacham S, Festuccia C. Nucleo-cytoplasmic transport as a therapeutic target of cancer. J Hematol Oncol. 2014;7:85.

18. Etchin J, Sun Q, Kentsis A, Farmer A, Zhang ZC, Sanda T, et al. Antileukemic activity of nuclear export inhibitors that spare normal hematopoietic cells. Leukemia. 2013;27:66-74.

19. Chen C, Siegel D, Gutierrez M, Jacoby M, Hofmeister CC, Gabrail N, et al. Safety and efficacy of selinexor in relapsed or refractory multiple myeloma and Waldenstrom's macroglobulinemia. Blood. 2018;131:855-63.

20. Gandhi UH, Senapedis W, Baloglu E, Unger TJ, Chari A, Vogl D, et al. Clinical implications of targeting XPO1-mediated nuclear export in multiple myeloma. Clin Lymphoma Myeloma Leuk. 2018;18:335-45.

21. Machlus KR, Wu SK, Vijey P, Soussou TS, Liu Z-J, Shacham E, et al. Selinexor-induced thrombocytopenia results from inhibition of thrombopoietin signaling in early megakaryopoiesis. Blood. 2017;130:1132-43.

22. Kapur R, Aslam R, Speck ER, Rebetz JM, Semple JW. Thrombopoietin receptor agonist (TPO-RA) treatment raises platelet counts and reduces anti-platelet antibody levels in mice with immune thrombocytopenia (ITP). Platelets. 2019;1-4 [Epub ahead of print]. 
23. Bunin DI, Bakke J, Green CE, Javitz HS, Fielden M, Chang PY. Romiplostim (Nplate ${ }^{\circ}$ ) as an effective radiation countermeasure to improve survival and platelet recovery in mice. Int $\mathbf{J}$ Radiat Biol. 2019;91:1-10.

24. Navari RM, Brenner MC. Treatment of cancer-related anorexia with olanzapine and megestrol acetate: a randomized trial. Support Care Cancer. 2010;18:951-6.

25. Vogl DT, Dingli D, Cornell RF, Huff CA, Jagannath S, Bhutani $\mathrm{D}$, et al. Selective inhibition of nuclear export with oral selinexor for treatment of relapsed or refractory multiple myeloma. J Clin Oncol. 2018;36:859-66. JCO2017755207.

26. Chari A, Vogl DT, Gavriatopoulou M, Nooka AK, Yee AJ, Huff $\mathrm{CA}$, et al. Oral selinexor-dexamethasone for triple-class refractory multiple myeloma. N Engl J Med. 2019;381:727-38.

27. Bahlis NJ, Sutherland H, White D, Sebag M, Lentzsch S, Kobt R, et al. Selinexor plus low-dose bortezomib and dexamethasone for patients with relapsed or refractory multiple myeloma. Blood. 2018;132:2546-54.

28. National Comprehensive Cancer Network (NCCN). Guidelines in oncology: antiemesis (Version 1.2019-February 29, 2019).

29. Roila F, Molassiotis A, Herrstedt J, Aapro M, Gralla RJ, Bruera E, et al. 2016 MASCC and ESMO guideline update for the prevention of chemotherapy- and radiotherapy-induced nausea and vomiting and of nausea and vomiting in advanced cancer patients. Ann Oncol. 2016;27 (Suppl 5) :v119-33.

30. Hing ZA, Fung HYJ, Ranganathan P, Mitchell S, El-Gamal D, Woyach JA, et al. Next-generation XPO1 inhibitor shows improved efficacy and in vivo tolerability in hematological malignancies. Leukemia. 2016;30:2364-72.

31. Bobillo S, Abrisqueta P, Carpio C, Raheja P, Castellvi J, Crespo $\mathrm{M}$, et al. Promising activity of selinexor in the treatment of a patient with refractory diffuse large B-cell lymphoma and central nervous system involvement. Haematologica. 2018;103:e92-3.

32. Lassman AB, Wen PY, Van Den Bent MJ, Plotkin SR, Walenkamp AME, Huang X, et al. Efficacy and safety of selinexor in recurrent glioblastoma. J Clin Oncol. 2019;37:2005.

33. National Comprehensive Cancer Network (NCCN). Guidelines in oncology: cancer related fatigue (Version 1.2019-March 12, 2019).

34. Lapolombella R, Sun Q, Williams K, Tangeman L, Jha S, Zhong Y, et al. Selective inhibitors of nuclear export show that CRM1/XPO1 is a target in chronic lymphocytic leukemia. Blood. 2012;120:4621-34.

35. Song X, Cong Z, Wilson K. Real-world treatment patterns, comorbidities, and disease-related complications in patients with multiple myeloma in the United States. Curr Med Res Opin. 2016;32:95-103.

\section{Affiliations}

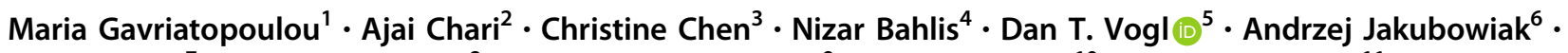
David Dingli $^{7} \cdot$ Robert F. Cornell $^{8} \cdot$ Craig C. Hofmeister $^{9}{ }^{9}$. David Siegel $\mathbb{1}^{10}$ - Jesus G. Berdeja ${ }^{11}$ - Donna Reece ${ }^{3}$. Darrell White ${ }^{12}$. Suzanne Lentzsch ${ }^{13} \cdot$ Cristina Gasparetto $^{14} \cdot$ Carol Ann Huff ${ }^{15} \cdot$ Sundar Jagannath $^{2} \cdot$ Rachid Baz $^{16}$. Ajay K. Nooka $\mathbb{1}^{17} \cdot$ Joshua Richter $^{10} \cdot$ Rafat Abonour $^{18} \cdot$ Terri L. Parker $^{19} \cdot$ Andrew J. Yee $^{20} \cdot$ Philippe Moreau $^{21}$. Sagar Lonial ${ }^{17} \cdot$ Sascha Tuchman $^{22} \cdot$ Katja C. Weisel $^{23} \cdot$ Mohamad Mohty $^{24} \cdot$ Sylvain Choquet $^{25} \cdot$ T. J. Unger ${ }^{26}$. ${\mathrm{Kai} \mathrm{Li}^{26} \cdot \mathrm{Yi} \text { Chai }}^{26} \cdot$ Lingling $\mathrm{Li}^{26} \cdot$ Jatin $\mathrm{Shah}^{26} \cdot$ Sharon Shacham ${ }^{26} \cdot$ Michael G. Kauffman ${ }^{26}$. Meletios Athanasios Dimopoulos ${ }^{1}$

1 Alexandra Hospital, School of Medicine, National and Kapodistrian University of Athens, Athens, Greece

2 Tisch Cancer Institute, Icahn School of Medicine at Mount Sinai, New York, NY, USA

3 Division of Medical Oncology \& Hematology, Princess Margaret Cancer Centre, Toronto, ON, Canada

4 Charbonneau Cancer Research Institute, Calgary, AB, Canada

5 Abramson Cancer Center, Perelman School of Medicine, University of Pennsylvania, Philadelphia, PA, USA

6 University of Chicago Medical Center, Chicago, IL, USA

7 Mayo Clinic, Rochester, MN, USA

8 Vanderbilt University Medical Center, Nashville, TN, USA

9 Department of Internal Medicine, Division of Hematology, The Ohio State University, Columbus, OH, USA

10 Department of Hematology, John Theurer Cancer Center, Hackensack, NJ, USA

11 Sarah Cannon Research Institute, Nashville, TN, USA

12 QEII Health Sciences Center, Dalhousie University, Halifax, NS, Canada
13 Department of Medicine, Division of Hematology/Oncology, Columbia University, New York, NY, USA

14 Duke University Cancer Center, Durham, NC, USA

15 Johns Hopkins University, Baltimore, MD, USA

16 Department of Malignant Hematology, H. Lee Moffitt Cancer Center and Research Institute, Tampa, FL, USA

17 Winship Cancer Institute, Emory University, Atlanta, GA, USA

18 Indiana University Cancer Center, Indianapolis, IN, USA

19 Yale School of Medicine, New Haven, CT, USA

20 Massachusetts General Hospital Cancer Center, Boston, MA, USA

21 University of Nantes, Nantes, France

22 Lineberger Comprehensive Cancer Center, University of North Carolina, Chapel Hill, NC, USA

23 University Medical Center of Hamburg-Eppendorf, Hamburg, Germany

24 Hôpital Saint Antoine, Paris, France

25 La Pitié-Salpêtrière Hospital, Paris, France

26 Karyopharm Therapeutics, Newton, MA, USA 